\title{
Consensus in Surgical Practice during the COVID-19 Pandemic: An Appraisal of the Literature
}

\author{
Ravindri Jayasinghe $^{1}$, Umesh Jayarajah ${ }^{1}$, Sanjeewa Seneviratne ${ }^{1}$ \\ ${ }^{1}$ Department of Surgery, Faculty of Medicine, University of Colombo, Sri Lanka
}

Key Words: surgery, surgical practice; COVID-19, pandemic, SARS-CoV-2, review, consensus, guidelines

\section{Correspondence to:}

Umesh Jayarajah, Email: umeshe.jaya@gmail.com

AND

Sanjeewa Seneviratne, Email: sanjeewa@srg.cmb.ac.lk

Department of Surgery, Faculty of Medicine, University of Colombo, Sri Lanka

Address: Department of Surgery, Faculty of Medicine, P.O. Box 271, Kynsey Road, Colombo 8, Western Province, Sri Lanka

Telephone: $\quad+94112671846$

\begin{abstract}
The coronavirus disease-2019 (COVID-19) pandemic is an ongoing pandemic caused by severe acute respiratory syndrome-coronavirus-2 (SARS-CoV-2). Although COVID-19 pandemic is not a direct surgical problem, its impact on the surgical units has been substantial. Many operations have been cancelled or deferred due to risk of disease spread and staff shortages. Operating theatres are identified as areas of high risk of disease transmission due to aerosol generation during the surgical procedures and prolonged patient contact. Resource limitations, including lack of personal protective equipment (PPE) and limited availability of testing, continue to expose the surgical community to COVID-19. In this review, evidence and consensus guidelines on surgical practice during the COVID-19 pandemic are summarised and described. Immediate action is deemed essential to ensure unhindered provision of surgical care while optimizing the use of limited resources and ensuring staff safety.
\end{abstract}




\section{Introduction and Background}

In late December 2019 hospitals in Wuhan, China reported an outbreak of a pneumonia of an unknown cause (Borges do Nascimento, Cacic, \& Abdulazeem, 2020). Later in January 2020, a novel coronavirus strain (SARS-CoV-2) was isolated from the suspected patients. Despite the early recognition and the public health measures, the infection spread rapidly across China and then the rest of the world. The World Health Organization (WHO) declared the outbreak as a Public Health Emergency of International Concern (PHEIC) on January $30^{\text {th }}$ 2020. As of $31^{\text {st }}$ May 2020,there were 5,934,936 confirmed cases of COVID-19 and 367,166 reported deaths worldwide (World Health Organization, 2020a). As a global control measure, governments enforced strict quarantine measures by placing most of the world population into a lockdown (Borges do Nascimento et al., 2020). Keeping with the recommendations of the Centres for Disease Control and Prevention (CDC), strict measures of social distancing were implemented while discouraging public gatherings which include work from home, closing of educational institutions and switching to alternative modes of teaching (Borges do Nascimento et al., 2020; Centers for Disease Control and Prevention, 2020c).

Although COVID-19 is not a primary surgical problem, its impact on the surgical units have been considerable, which to some extent could be viewed as a mass casualty incident with a global impact. With human and financial resources overwhelmed, the rational use of available surgical resources has become a matter of utmost importance. While the virus continues to create havoc across medical systems, the main threats to the surgical community are related to; staff management, halting elective procedures, effective triaging of overwhelmed resources, practicing precautions to combat high risk of transmission and social distancing at the workplace. During the COVID-19 crisis, applications of strengths learnt in managing trauma, critical care, and triage and extrapolate them into practice, would help the nations may combat this catastrophe effectively. The objective of this review is to summarize and describe the current evidence and consensus guidelines on surgical practice during the COVID-19 pandemic. 


\section{Review}

\section{Preoperative preparation and general precautions}

Managing logistics in order to provide optimum patient care should be the goal in all surgical centres. In scheduling patients for surgery, evaluating the individual patient's risks of infection versus consequences due to delay in procedure should be considered. During the decision making, it is also important to consider the availability of resources such as PPE, staff and other medical and logistical concerns (American College of Surgeons, 2020c). Elective cases should be triaged balancing the clinical judgement on urgency of the surgery with the availability of resources. This may also include non-surgical management whenever such alternatives are available which do not significantly alter patient outcomes (American College of Surgeons, 2020c). The Royal College of Surgeons of England recommends considering the logistical capacity determined by the theatre manager, the risk to patient outcomes due to the delay determined by a surgeon in the relevant speciality balanced with the capacity of the institution to manage an outbreak. This triage should be based on a real time data driven assessment (Royal College of Surgeons of England, 2020).

Wearing adequate PPE should be considered in all situations involving patients confirmed or suspected to have COVID-19. The American College of Surgeons has published a set of guidelines on the use of PPE in the operating room (OR) in line with the CDC recommendations for the use of PPE for healthcare personnel (American College of Surgeons, 2020e; Centers for Disease Control and Prevention, 2020a). In addition to asymptomatic carriers of COVID-19, as the incubation period of the virus may be as long as 14 days, there can be asymptomatic infected individuals posing a threat to the health care personnel. It is recommended to wear N95 respirators in the OR since standard surgical facemasks will not protect against virus transmission from aerosol generating procedures. Depending on the logistic constraints, institutions may implement policies on extended use or limited reuse of N95 masks so that adequate supplies would be available at times of peak demand. Such limitations may additionally require minimizing the individuals needing respiratory protection, using alternatives where feasible, and prioritizing the personnel having the highest risk of exposure as recommended by the CDC and the American Society of Anaesthesiologists (American Society of Anesthesiologists, 2020; Centers for Disease Control and Prevention, 2020a). 
The Secretary General of the WHO emphasized on the importance of testing during the pandemic where he said "You cannot fight a fire blindfolded. Our key message is test, test, test" (World Health Organization, 2020b). Therefore, it is important to consider pre-operative testing of patients undergoing surgery. Testing for COVID-19 may be limited to certain categories of patients in some countries due to the scarcity of resources and labs. Priority needs to be given to patients having suspicious symptoms, evidence of respiratory illness, high risk due to contact history and in patients from areas of outbreaks. Although, surgeons are encouraged to request testing in patients scheduled for surgery, this has to be balanced with capacity for COVID-19 testing and potential risk of COVID-19 in the community. The available tests for COVID-19 include nucleic acid amplification tests for viral RNA using reverse transcription-polymerase chain reaction (RT-PCR) and serology for antibody detection which includes detection of $\operatorname{IgM}, \operatorname{IgA}, \operatorname{IgG}$ or total antibodies (Al-Muharraqi, 2020). Studies have suggested that most COVID-19 patients seroconvert within seven to eleven days although, some may develop antibodies sooner. Even though antibody testing may not be of much use for diagnosis, it may be used to identify individuals who were previously infected and recovered as well as individuals who may be immune to COVID-19 (Al-Muharraqi, 2020). Regardless, until reliable and cost-effective widespread testing becomes available, it is advisable to consider all patients as positive for COVID-19 until proven otherwise.

Hospital environment itself may spread the disease to patients awaiting surgery, who in turn may spread the disease among other patients and healthcare staff. This risk will be greater among patients with prolonged hospital stays due to potential cancellations leading to delays in surgery. Therefore, ideally all patients should be screened with the gold standard test (RTPCR) 24 hours before the procedure with or without an antibody test followed by isolation until surgery (Al-Muharraqi, 2020). If they test positive for PCR, public health protocols for COVID-19 should be followed and the surgery is postponed in case of routine surgeries. If positive for only the antibodies they will require further testing to confirm complete recovery from COVID-19. If they are negative for both the PCR and the antibody screen, still they may require weekly PCR during the hospital stay and be discharged after undergoing a PCR for confirmation (Al-Muharraqi, 2020). Although these are necessary for optimum management, such repeated investigations would be a major burden to many resource limited settings in developing as well as developed countries during the height of the pandemic. 
Forrester et al, have developed an algorithm on precautions for OR based on urgency of the surgery, expected viral load, and the likelihood of aerosolization of the virus in case of a suspected patient based on symptoms and testing. Further, they have recommended assuming all patients undergoing emergency procedures to be infected and appropriate safety precautions to be practiced. All aerosol generating procedures of the upper aero-digestive tract as well as endoscopy, open or laparoscopic bowel surgery are included into the high risk category, given that the virus may survive in the gastrointestinal tract (Chiu et al., 2020; Forrester, Nassar, Maggio, \& Hawn, 2020). As aerosolization of the virus may occur through exhaled gases, it is advised that even when a procedure is performed under deep sedation, to avoid use of high flow oxygen and if feasible the patient to wear a surgical face mask throughout the procedure (Rajan \& Joshi, 2020). Similar to the testing algorithm discussed above, if patients were found to be symptomatic, surgery should be delayed in non-urgent cases. Such patients are considered for RT-PCR only if there is a significant risk to the patient in delaying the surgery. Once the patient is proven to be positive, after the approval of the surgical and the anaesthetic teams, the surgery could proceed with precautionary measures with all members of the teams wearing appropriate protective attire as recommended by the CDC. For patients who test negative by RT-PCR, the team members would use standard surgical attire. Face shields and N95 respirators are recommended to be worn during suspected aerosol generating procedures. All cleaning personal of the OR are advised to follow droplet precautions as a safety measure (Centers for Disease Control and Prevention, 2020a; Forrester et al., 2020).

Practicing social distancing and avoiding public gatherings at the workplace should also be practiced as recommended by the CDC to contain the possible spread of the virus within the working environment (Centers for Disease Control and Prevention, 2020c). Such measures help increase the doubling time of the virus, thus controlling the spread. Segregating staff between hospitals is also an important measure which would also prevent cross infection between hospitals. Resorting to web-conferencing instead of large meetings is recommended and should be implemented. Taking staff attendance at small group meetings would further help contact tracing in the event of an outbreak (Wong et al., 2020). Furthermore, it is important to develop a pandemic preparedness protocol in collaboration with surgeons and anaesthesiologists with the involvement of infection control experts which should be incorporated into the routine hospital management plan. Staff training should be considered an immediate priority and should be done well ahead of perceiving the threat with effective 
communication between the hospital's central command team and the surgical teams (Collaborative, 2020). In addition to these, Brindle et al based on their experience, have suggested several other precautionary measures to manage surgical systems during the pandemic (Brindle \& Gawande, 2020). These include having a clear plan in place for essential surgeries (life and limb saving surgeries and cancer surgery where timely intervention decreases morbidity and mortality), limiting the theatre staff only to the essential number and having a dedicated operating area for suspected COVID-19 patients (Brindle \& Gawande, 2020). These precautionary measures are aimed at reducing the spread of infection and preventing the staff from acquiring the infection which would further decrease the capacity of the healthcare system.

\section{Theatre setting during the COVID-19 pandemic}

Due to the highly contagious nature of the virus and its spread through fomites, it is necessary to have designated ORs for COVID-19 patients undergoing surgery. Choosing an OR in close proximity to the entrance of the theatre block would minimize the potential for contamination of other theatres (Coccolini et al., 2020). Patient transport to the theatre block is recognized to be associated with a high risk of disease transmission. It is recommended to have a short predetermined path for transport, avoiding any public areas and areas with non-infected patients as much as possible. The personnel transporting the patient should be properly equipped with adequate PPE and should have had training in handling high risk patients. Furthermore, the patient should wear a facemask ideally an N-95 as a precaution. The environmental surfaces coming in contact should be immediately sanitized before and after patient transport. It is useful to have a dedicated team to handle sanitization. Having the same personnel handle a single patient will be beneficial in effectively managing resources. Furthermore, bringing non-essential items including personal items to the OR should be avoided. All disposable items should be discarded after a single case, traffic in and out of the theatre should be minimised, a dedicated recovery area should be allocated for suspected patients, or patients may be recovered within the OR itself (Brindle \& Gawande, 2020). Where possible, the duration of the case could be shortened to decrease staff exposure (Brindle \& Gawande, 2020).

Standard ORs are designed to have a positive pressure environment. However, during the pandemic, it is ideal to have a negative pressure environment with a high ( 25 per hour) 
frequency of air changes which prevents dissemination of the virus from the OR. Measures should be taken to limit the flow of contaminated air out of the OR through only one outlet. With the use of a designated OR for suspected patients, a designated workflow should also be established with coordination of the staff, while allocating a separate anaesthesiologist for the designated OR with theallocation of a separate area for donning and doffing of PPE as recommended by the CDC (Centers for Disease Control and Prevention, 2020b).

Furthermore, strict infection control practices and decontamination procedures including routine checks and cleaning of the anaesthetic machines and powered air purifying respirator (PAPR) sets are necessary (Wong et al., 2020). Where feasible, it is advised to discard the anaesthetic breathing circuit and the canister of soda lime in order to avoid potential cross contamination (T. Tan, 2004). High-efficiency particulate air filters (HEPA) should be used in the anaesthetic machine at the patient's end and also between the anaesthetic machine and the expiratory limb of the circuit (Wong et al., 2020). Single use equipment should be used where possible in the OR and only a minimum amount of equipment and drugs should be bought to the premises which reduces the number of equipment needing cleaning (T. Tan, 2004; Tompkins \& Kerchberger, 2010). The surfaces of electronic appliances coming into frequent contact with staff can be covered with plastic wrap for the ease of decontamination (Wong et al., 2020).

Measures should be taken to minimize the risk of spread of infection among the staff. This include risk assessment and serology testing of members of the teams working in the OR and limiting the number of staff in the OR by having smaller teams than usual who would work with proper coordination (Brücher et al., 2020). Additionally, surgical sections should be ideally divided into two categories, a dedicated section for positive patients which only needs minimum OR and surgical staff and another section dedicated for emergency surgery and urgent oncological surgery and depending on the risk assessment, for other routine surgeries in COVID-19 negative patents (Zheng, Boni, \& Fingerhut, 2020).

Environmental sanitization, proper waste disposal and linen management contribute substantially for infection control within the facility. After every procedure the OR and its surroundings should be adequately sanitized including the areas where COVID-19 patients have transited. Maintaining hygiene should be the responsibility of all the individuals of the team. Single use contaminated material should be discarded, and reusable material should be decontaminated and disinfected conforming to the institutional protocols. Wearing full PPE 
during sanitizing procedures is a must. A dedicated area should be assigned for disposal of waste, and appropriate PPE should be worn in transporting and handling such waste. Linen can also be contaminated and therefore, should be handled with proper care wearing PPE. They should not be placed on floors but in dedicated containers and immediately sent for cleaning minimizing the time left outside the OR (Coccolini et al., 2020). The cleaning of surgical equipment used in procedures of a COVID-19 positive or suspected patient should be done separately from other surgical equipment (Society of American gastrointestinal and endoscopic surgeons (SAGES), 2020).

\section{Resource management and triage}

Due to the scarcity of resources with the rising case load, triaging of patients will be required in addition to optimum preparedness to deal with all surgical patients efficiently and effectively. The surgical teams should consider their patients' surgical needs and the capacity of the team and the hospital in real time. The surgeons should take time to carefully review the elective procedures and minimise invasive procedures unless they are deemed essential. Where possible, inpatient diagnostic and surgical procedures should be shifted to outpatient settings (Royal College of Surgeons of England, 2020). A real time account of the available resources should be kept and the decisions taken based on this data. This includes assessing the number of occupied beds, the total number of admission and planned procedures and the number of available intensive care unit (ICU) beds. To maximise the available resources, double bedding of hospital and ICUs may be considered. With the rising demand, post anaesthesia care units, ORs can also be converted to ICU beds if necessary (Elster, Potter, \& Chung, 2020). Even though elective surgical procedures are postponed, medically necessary and time sensitive procedures should be directed to ambulatory surgery centres with no inpatient capacity which can be used to offload the facility, preserving resources (Elster et al., 2020). Use of such ambulatory surgical centres to offload the acute care facilities would make more ORs available, preserve supplies, and hospital staff to dedicate their time more towards handling the increasing case load. However, not all of these centres are suitable for shifting procedures that are typically performed in hospitals because of the need for specialised surgical equipment and intense monitoring during and after the procedure, and extensive in person follow-up which may exceed the scope of these centres. Even though some may not fulfil these demands, they can still be of help by providing available resources such as equipment and disposables (Rajan \& Joshi, 2020). Centres with the potential for 
receiving such offloading patients from acute care hospitals should have a solid plan of handling the inflow of patients with adequately trained staff and infection control measures.

COVID-19 positive patients are recommended not to undergo non-emergency operations as the pneumonia from COVID-19 carries a high rate of mortality especially during peri and post-operative period (Brücher et al., 2020). The Australian and New Zealand Hepatic, Pancreatic and Biliary Association (ANZHPBA) has provided a guide in triaging for the surgeons in three phases depending on the severity of the COVID-19 situation in the locality while considering the availability of the resources. Phase one is a semi urgent setting where institutional resources aren't exhausted and the ventilator capacity and the ICU capacity is still preserved, Phase two is an urgent setting with limited ICU and ventilator capacity, and phase three is when all hospital resources are routed to COVID-19 patients where supplies are exhausted. This guidance elaborates which procedures to consider with regards to hepatobiliary surgery during the pandemic depending on their urgency (Australian and New Zealand Hepatic, 2020). The intercollegiate general surgery guidelines on COVID-19 recommends giving priority to acute patients given that they are considered infected unless proven otherwise (Royal College of Surgeons of Edinburgh, 2020). Furthermore, nonsurgical management for all possible cases such as early appendicitis and acute cholecystitis is recommended. The use of open approach is preferred over laparoscopy in the same scenarios (Royal College of Surgeons of Edinburgh, 2020). Also procedures such as thoracoscopy and endoscopy should also be avoided as much as possible (American College of Surgeons, 2020c; Centers for Medicare \& Medicaid Services (CMS), 2020; Royal College of Surgeons of England, 2020).

With the emergence of technology-based alternatives, telemedicine could be used as a frontline triage for surgical clinics. However, the health insurance portability and accountability act (HIPAA) related concerns in countries like the USA will pose practical limitations in utilizing these platforms (Ross et al., 2020). Despite that many can be tailored according to the requirement of the individualized clinic scenario. Virtual visits and telephone screening triage may be useful alternatives depending on the burden (Ross et al., 2020). These techniques not only evaluate the patient's ongoing medical concerns but also will screen them for potential COVID-19 infection with the use of disease screening questions. If detected as high risk, they can be directed to COVID-19 testing. According to this method, the patients are classified into three categories based on their need; ideal to be 
evaluated by a phone call only, patient needing a virtual clinic visit and patient needing objective a physical evaluation with investigations. This is all possible through a designed virtual communication application. After evaluation via the virtual visit, the developed individualized management plan is documented with the patient's electronic medical record. Whenever a patient required a physical clinic visit, they are allowed in with only one caregiver after screening for potential COVID-19 symptoms. On arrival, they will again go through a screen for COVID-19 symptoms and if deemed high risk, are directed for testing (Ross et al., 2020). The National Institute for Health and Care Excellence (NICE) had issued guidance on steps to follow when admitting a patient to hospital when needing critical care, in order to maximise the safety of patients while ensuring staff safety. It emphasises on clinical decision making considering the likelihood of patient recovery, making the best use of critical care resources and precise coordination (National Institute for Health and Care Excellence, 2020).

With the increasing case burden from COVID-19, the hospital management will have to equip with a plan to handle a situation where all the hospital resources are exhausted, including staff due to quarantine, PPE, blood, ventilators, other essential consumables and space including hospital beds. During such a scenario, it is suggested that all non-emergent cases be cancelled, all transfers being overseen by an acute care surgeon, expanding ICU beds and staffing by deploying members from other teams including surgical teams. Finally in a situation like in Italy or Wuhan, China it will be necessary for the acute care surgeons to focus on the ICU patient care while non-acute care surgeons cover trauma, non-operative measures considered for most diseases and all non-COVID care being triaged (Ross et al., 2020).

In the United Kingdom the National Health Service (NHS) had ordered to suspend all elective surgeries from the $15^{\text {th }}$ April for a period of three months in order to expand critical care capacity to a maximum. By this measure, they expect to free up acute care beds making increasing the capacity available for managing the surge of COVID-19 patients. However this excludes emergency admissions, cancer treatment and other urgent operations (Iacobucci, 2020). Lessons should be learnt from Italy, that a pandemic should not be considered lightly at any point. In a globalized world, an epidemic can become a pandemic with a single airline flight no matter how distant the outbreak may seem. Taking measures in advance would help save millions of lives as the saying "prevention is better than cure". 
Another fact that goes unnoticed is the psychological distress the staff will undergo with critical incident stress syndrome with emotional reactions compromising private and social life(Guerci et al., 2020). This may lead to reduced work efficiency further contributing to already exhausted manpower.

\section{Elective surgery during the COVID-19 pandemic}

During the pandemic, most of the frontline healthcare workers were forced to alter their professional responsibilities in order to meet the hospital needs. General surgeons seemed mostly affected due to the diverse procedures they perform. Due to the impact of the pandemic on the health system, surgeons witnessed a dramatic change in their practice with decreasing elective surgeries. Many countries have imposed regulations limiting elective surgeries in order to make their acute care facilities function at maximum capacity (Iacobucci, 2020; "State guidance on elective surgeries," 2020). Despite the frequently changing guidelines, individual decisions should be taken considering the situation in the locality and consulting an expert panel not limited to surgeons but including nurses, resource managers, hospital administration and epidemiologists. Furthermore, it is important to identify the trajectory of the local COVID-19 disease in guiding these recommendations (Diaz, Sarac, Schoenbrunner, Janis, \& Pawlik, 2020). Although challenging, it is important to strike a balance in managing the available resources such as PPE, staffing, beds, equipment with the urgency and time sensitivity of the patient's condition in order to provide the best timely care to the patient.

A joint guideline had been released with the collaboration of the American College of Surgeons, American Society of Anaesthesiologists, Association of Perioperative Registered Nurses, and the American Hospital Association on resuming elective surgery after the COVID-19 pandemic in the USA. This elaborates on the management of the pent-up patient demand for surgical care after recommencing elective surgery. The timing of reopening should be after a sustained reduction of the rate of detecting new COVID-19 cases with the hospitals are no longer using crisis standards of care. They also should have adequate staff, ICU beds, PPE, ventilators and other medical supplies. Patient and staff testing should be freely available and proper protocols should be in place for handling positive patients and staff. Prioritization of cases should be done with a scoring system with identification of essential health care staff for the procedures and their willingness to comply with the 
increased workload. Strategies should be in place for phased opening of ORs after identifying the capacity goals. Further, the guidance recommends having institutional guidelines for the five phases of surgical care; preoperative, immediate preoperative, intraoperative, postoperative and post discharge care planning ensuring the safety of patients and staff. It also emphasises on keeping records in order to reflect and re-evaluate. It is further recommended to expect and be ready for a possible second wave of infection and have plans in hand for risk mitigation (American College of Surgeons, 2020d).

\section{Recommendations for individual disciplines}

Guidance had been released from many surgical societies globally on their relevant disciplines emphasizing on handling the surgical needs of patients during the pandemic. When the hospital management and the governments are focusing on conserving ICU beds, ventilators, PPE, etc., the decisions taken to postpone elective and semi-elective surgery, may increase the morbidity and mortality of many patients awaiting such procedures.

Cancer surgery is one such speciality. According to the American College of Surgeons, the guiding principles in pursuing with surgery should be resource considerations and cancer care coordination. Individual resources at an institutional level should be considered assessing the preparedness of the facility. Due to the elective cases especially the ones which have a higher probability of requiring ICU beds and ventilators, it becomes necessary to balance the risk of delay of the individual patient with the availability of these resources for patients affected by COVID-19. Shared decision making should be done and the priority of the case should be assessed with a multi-disciplinary team. Triage guidance for cancer patients is presented in three phases similar to the ANZHPBA guidelines discussed above. In general phase one, describes a semi urgent setting where resources and the ICU capacity are not exhausted. In During this phase, surgery is recommended in patients likely to have compromised survival if surgery is not performed within three months. In phase two, in a setting with many COVID19 patients with limited resources, surgery is recommended in patients who will have compromised survival if not performed within a few days. In phase three, where all resources in the hospital are routed to COVID-19 patients, surgery is recommended in patients where survival is compromised if surgery is not performed within a few hours (American College of Surgeons, 2020a). The European Society of Medical Oncology (ESMO) has emphasised on the challenging need to manage patients with cancer optimally during COVID-19 pandemic 
as data from Italy had revealed that $20 \%$ of the deaths were among patients with active cancer (European Society for Medical Oncology (ESMO), 2020). Furthermore, it emphasises on the use of telemedicine in order to limit clinic visits and switching to oral or subcutaneous therapies when possible instead of intravenous therapy. It had also advised against seeing elderly patients over 70 years at the clinic setting (European Society for Medical Oncology (ESMO), 2020). Guidance from the NHS, United Kingdom suggests that out of the cancer patients, certain groups are more vulnerable for serious illness with COVID-19. Those include patients currently undergoing active chemotherapy or radical radiotherapy for lung cancer and patients with haematological malignancies due to the difference in the degree of immunosuppression (Boffa, 1920; National Health Service (NHS), 2020). However, other patients undergoing cancer treatment may have treatment related immune suppression and therefore as a group, these patients are at a higher risk. The NHS guidance had set priority levels for various modalities of cancer treatment. For example, there are six levels of priority for systemic treatment, and five levels of priority for radiotherapy (National Health Service (NHS), 2020). Considering the lessons learned from China and Italy it is important to prioritise the needs and the risks of these patients and take informed decisions with the patient's best interest in mind (Burki, 2020).

The Society of American Gastrointestinal and Endoscopic Surgeons (SAGES) had put forward guidance in practice in order to protect patients, surgeons and the staff from infection. Keeping with the recommendations of the other scientific societies, they had also recommended postponing all elective surgical and endoscopic cases depending on the local guidelines. Furthermore, it supports postponing non-urgent clinic visits and switching to telemedicine and letting all non-essential hospital staff work from home. Also, it recommends resorting to technological alternatives to hold multidisciplinary meetings. Even though evidence has shown the possibility of aerosolization of blood born viruses with laparoscopy, it was not proven so far for COVID-19 in particular. Regardless, precautionary measures should be taken when attempting minimally invasive surgery and laparoscopy in suspected patients. It also emphasises on the risks to endoscopists and airway procedures and therefore, strict use of PPE is advised. The use of monopolar electrosurgery, ultrasonic dissectors and advance bipolar devices should be minimised due to the risk of particle aerosolization. The main risk in laparoscopic surgery is, if the pathogen is present in the peritoneal cavity and the aerosol is released, it would contaminate the OR during surgery and during exsufflation after surgery (Tuech et al., 2020; Zheng et al., 2020). Therefore, during laparoscopic surgery, 
smallest possible ports are used to minimise leakage around the port and $\mathrm{CO}_{2}$ insufflation pressure is advised to be kept to a minimum with the use of ultra-filtration if possible (Society of American gastrointestinal and endoscopic surgeons (SAGES), 2020).

As a part of the team in the OR, anaesthesiologists also face major hazards due to procedures involving with a high risk of aerosol generation. Careful prior planning and necessary safety precautions are taken in order to ensure personal safety while ensuring optimum care. Endotracheal intubation is preferred to non-invasive ventilation in patients with COVID-19. During intubation, it is recommended to use disposable airway equipment and the staff be equipped with adequate PPE with Filtering Face Piece-3 (FFP-3) filters. It is best to use a technique which is most likely to be successful at its first attempt. Fiberscope intubation has risks associated with aerosolization and therefore should be best avoided. Rapid sequence intubation is considered to be better at minimizing aerosolization. Manual ventilation should be avoided at all times. If feasible, a dedicated ventilator should be used for COVID-19 positive patients in the OR (Coccolini et al., 2020). The use of an aerosol box is adopted in many ORs globally which shields the face of the provider during endotracheal intubation while they have the freedom to move their arms freely during intubating (Brücher et al., 2020). Anti-emetics should be administered routinely to avoid post-operative retching. All equipment coming into contact with patients' secretions should be carefully handled. The patient should wear a surgical mask following extubation. Nasal prongs underneath the mask may be used to provide supplemental oxygen but the use of venturi masks for this purpose should be avoided due to the potential risk of aerosolization of the virus (Wong et al., 2020).

COVID-19 has made substantial changes in many surgical specialities. With regards to neurosurgery, risks have been identified with certain procedures such as trans-sphenoidal and endonasal surgery due to the risks associated with the mode of transmission of the virus. Therefore, these procedures are recommended to be replaced by alternative transcranial approaches (Patel et al., 2020). Any surgery needing a microscope is nearly impossible while wearing a PAPR. Hence, surgeries with high risk, for instance surgery which involves entering air sinuses should be performed with additional precautions (Amin-Hanjani et al., 2020). Emergency neurosurgery should be performed in patients presenting with life threatening conditions. It is also suggested that speed drilling the skull should be avoided to reduce the risk of skull bone aerosols. Furthermore, it is suggested to reduce the duration and the blood loss in surgery as much as possible (Y.-t. Tan et al., 2020). With regards to 
otolaryngologists, there are certain specific concerns as they often work in the upper aerodigestive tract. In most places, all elective procedures that would aerosolize tissue including tonsillectomy, mastoid drilling, sinus surgery and other airway procedures have been deferred (Vukkadala, Qian, Holsinger, Patel, \& Rosenthal, 2020). COVID-19 patients may require tracheostomy when prolong ventilation is required. If a need arises, it is suggested to follow precautions to minimise the aerosolization of the virus such as complete paralysis to avoid coughing, ventilation only after cuff inflation, stopping ventilation before entering the airway and minimising suctioning and cautery. During post-tracheostomy, it is recommended to keep the cuff inflated, to use in-line suction, and delay tube change until the risk of COVID-19 spread is minimal (Vukkadala et al., 2020).

\section{Emergency surgery during the COVID-19 pandemic}

Emergency surgical care during the pandemic could be unrelated or directly related to COVID-19. In one hand, emergency surgery may be needed in patients admitted with COVID-19, especially in the ICU they are prone to develop mesenteric ischemia, perforation of hollow viscera and conditions like acute limb ischemia (Collaborative, 2020). On the other hand, there may be patients with acute surgical problems unrelated to COVID-19 requiring emergency surgery who may or may not have concurrent COVID-19 infection (Collaborative, 2020). In emergency setup, testing all patients for COVID-19 before surgery is ideal and all patients should be assumed positive unless proven otherwise (Brücher et al., 2020).

According to the guidelines by the American College of Surgeons, emergency surgical procedures should be undertaken if delaying would likely prolong hospital stay or cause potential harm to the patient. Furthermore, it states that certain surgical conditions may be managed non-operatively or if necessary in an outpatient setting if possible. These include acute thrombosed haemorrhoids, superficial perianal abscesses, superficial and localised soft tissue infections, uncomplicated appendicitis and symptomatic cholelithiasis. It is advisable to opt for non-surgical interventions (i.e. radiological) in conditions such as acute pancreatitis complicated with necrosis while emergency surgery is mandated in conditions including bowel perforation, intestinal ischemia, and strangulated hernias(American College of Surgeons, 2020b). 
Despite the threats posed by the pandemic, trauma patients will require urgent lifesaving interventions. This pandemic has hindered the ability of trauma care centres to provide care for the critically injured. Guidelines have been issued on the management for trauma centres during the pandemic. The American College of Surgeons committee on trauma had proposed a planning process which involves regional planning, hospital planning involving the considerations on ICU capacity and capabilities and training of staff ensuring protection of both staff and patients. Furthermore, they suggest strategies that should be implemented at the point of care. The trauma bay should determine a patient's COVID-19 status by an assessment of their symptoms and, droplet and contact precautions should be followed for all patients. The OR should work based on the hospital policy for COVID-19 patients preventing any delays. The hospital should be well aware of its ICU capacity and the availability of ventilators. Proper management of scarce resources such as blood products and PPE should be done, and hospital policies should be implemented in preserving these. Setting up restrictive transfusion strategies in the ICUs for blood products and encouraging blood donation in the community should be done to conserve and ensure the availability of blood (American College of Surgeons Committee on Trauma, 2020).

\section{Complications and challenges}

Deciding on surgery in a patient during the pandemic is a challenge. In the light of COVID19 , if a patient develops fever and pulmonary complications postoperatively while recovering from elective surgery, diagnostic testing become mandatory in order to exclude COVID-19 and if becomes positive, for contact tracing. Ideally, screening all patients undergoing elective surgery with RT-PCR should be done (Aminian, Safari, Razeghian-Jahromi, Ghorbani, \& Delaney, 2020). Studies during the early stages of the epidemic in Wuhan, China in patients undergoing major surgery with COVID-19 during the peri-operative period have shown a high incidence of major complications with extremely high mortality (Lei et al., 2020). Patients who develop post-operative fever or having pyrexia of unknown origin or respiratory symptoms should be sent for isolation and RT-PCR or serology testing be performed with or without a computed tomography of the chest. It is important to deploy COVID-19 specific surgical teams to care for suspected patients where possible. Before discharge from the hospital, testing should be done and if they are still infectious, they should be sent to quarantine to minimize the spread (Collaborative, 2020). During admission, as a 
measure to minimize the spread and unnecessary exposure, routine postoperative visits can be replaced with phone calls and virtual visits where feasible (Wong et al., 2020).

\section{Conclusions}

The COVID-19 pandemic had caused a paradigm shift in the health sector. It has made a dramatic impact in the professional commitments of surgeons in their diverse specialities. Preventing infection in a high-risk work setting and providing optimum care to the patients by managing limited available resources in the facility is challenging. In order to face this challenge, modifications in infrastructure, strict healthcare policy changes and solid infection control strategies needs to be implemented. This should be done with the involvement of multiple stakeholders including surgeons, anaesthetists, nurses and support staff. Aligning interests of all parties is essential in providing unhindered care at the patients' best interest minimising the risk of viral transmission to other patients and healthcare workers.

\section{List of abbreviations}

COVID-19: Coronavirus disease-2019

SARS-CoV-2: Severe acute respiratory syndrome-coronavirus-2

WHO: World Health Organization

PHEIC: Public Health Emergency of International Concern

CDC: Centres for Disease Control and Prevention

RT-PCR: Reverse Transcription -PCR Polymerase Chain Reaction

OR: Operating Room

HEPA: High-efficiency particulate air filters

PAPR: Powered Air Purifying Respirator

PPE: Personal Protective Equipment

ICU: Intensive Care Unit

ANZHPBA: Australian and New Zealand Hepatic, Pancreatic and Biliary Association

HIPPA: Health Insurance Portability and Accountability act

NICE: National Institute for Health and Care Excellence

NHS: National Health Services

ESMO: European Society of Medical Oncology

SAGES: Society of American Gastrointestinal and Endoscopic Surgeons 
FFP3: Filtering Face Piece-3

USA: United States of America

UK: United Kingdom

\section{Additional Information}

\section{Disclosures}

Conflicts of interest: In compliance with the ICMJE uniform disclosure form, all authors declare the following: Payment/services info: All authors have declared that no financial support was received from any organization for the submitted work. Financial relationships: All authors have declared that they have no financial relationships at present or within the previous three years with any organizations that might have an interest in the submitted work. Other relationships: All authors have declared that there are no other relationships or activities that could appear to have influenced the submitted work.

\section{References}

Al-Muharraqi, M. (2020). Testing recommendation for COVID-19 (SARS-CoV-2) in patients planned for surgery-continuing the service and 'suppressing'the pandemic. The British Journal of Oral \& Maxillofacial Surgery.

American College of Surgeons. (2020a). COVID-19 Guidelines for Triage of Cancer Surgery Patients. Retrieved May, 2020, from https://www.facs.org/covid-19/clinicalguidance/elective-case/cancer-surgery

American College of Surgeons. (2020b). COVID-19: Elective Case Triage Guidelines for Surgical Care. Retrieved May, 2020, from https://www.facs.org/covid-19/clinicalguidance/elective-case

American College of Surgeons. (2020c). COVID-19: guidance for triage of non-emergent surgical procedures. Retrieved May, 2020, from https://www.facs.org/covid19/clinical-guidance/triage

American College of Surgeons. (2020d). Joint Statement: Roadmap for Resuming Elective Surgery after COVID-19 Pandemic. Retrieved May, 2020, from https://www.facs.org/covid-19/clinical-guidance/roadmap-elective-surgery

American College of Surgeons. (2020e). Other PPE recommendations. Retrieved May, 2020, from https://www.facs.org/covid-19/ppe/additional.

American College of Surgeons Committee on Trauma. (2020). Maintaining Trauma Center Access \& Care during the COVID-19 Pandemic: Guidance Document for Trauma Medical Directors. Retrieved May, 2020, from https://www.facs.org/qualityprograms/trauma/maintaining-access

American Society of Anesthesiologists. (2020). UPDATE: The Use of Personal Protective Equipment by Anesthesia Professionals during the COVID-19 Pandemic. Retrieved May, 2020, from https://www.asahq.org/about-asa/newsroom/news- 
releases/2020/03/update-the-use-of-personal-protective-equipment-by-anesthesiaprofessionals-during-the-covid-19-pandemic

Amin-Hanjani, S., Bambakidis, N. C., Barker, F. G., Carter, B. S., Cockroft, K. M., Du, R., . . . Jane, J. A. (2020). COVID-19 and neurosurgical practice: an interim report. Journal of Neurosurgery, 1 (aop), 1-2.

Aminian, A., Safari, S., Razeghian-Jahromi, A., Ghorbani, M., \& Delaney, C. P. (2020). COVID-19 outbreak and surgical practice: unexpected fatality in perioperative period. Annals of surgery.

Australian and New Zealand Hepatic, P. a. B. A. A. (2020). Considerations for HPB Surgeons in a Complex Triage Scenario COVID-19. Retrieved May, 2020, from https://www.anzhpba.com/wp-content/uploads/2020/04/Considerations-for-HPBsurgeons-in-a-complex-triage-scenario-COVID19_1.pdf

Boffa, D. J. (1920). COVID-19 Guidance for Triage of Operationn for Thoracic Malignancien: A Connennun Statement from Thoracic Surgery Outcomen Renearch Network.

Borges do Nascimento, I. J., Cacic, N., \& Abdulazeem, H. M. (2020). Novel Coronavirus Infection (COVID-19) in Humans: A Scoping Review and Meta-Analysis. 9(4). doi: 10.3390/jcm9040941

Brindle, M. E., \& Gawande, A. (2020). Managing COVID-19 in surgical systems. Annals of surgery.

Brücher, B. L., Nigri, G., Tinelli, A., Lapeña, J. F. F., Espin-Basany, E., Macri, P., . . Lück, R. (2020). COVID-19: Pandemic surgery guidance. 4open, 3, 1.

Burki, T. K. (2020). Cancer guidelines during the COVID-19 pandemic. The Lancet Oncology, 21(5), 629-630.

Centers for Disease Control and Prevention. (2020a). Interim Infection Prevention and Control Recommendations for Patients with Suspected or Confirmed Coronavirus Disease 2019 (COVID-19) in Healthcare Settings. Retrieved May, 2020, from https://www.cdc.gov/coronavirus/2019-ncov/infection-control/controlrecommendations.html?CDC_AA_refVal=https\%3A\%2F\%2Fwww.cdc.gov\%2Fcoro navirus\%2F2019-ncov\%2Fhcp\%2Finfection-control.html

Centers for Disease Control and Prevention. (2020b). Sequence for putting on personal protective eqipment. Retrieved May, 2020, from https://www.cdc.gov/hai/pdfs/ppe/PPE-Sequence.pdf

Centers for Disease Control and Prevention. (2020c). Social Distancing. Retrieved May, 2020, from https://www.cdc.gov/coronavirus/2019-ncov/prevent-getting-sick/socialdistancing.html

Centers for Medicare \& Medicaid Services (CMS). (2020). CMS Adult Elective Surgery and Procedures Recommendations: Limit all non-essential planned surgeries and procedures, including dental, until further notice. Retrieved May, 2020, from https://www.cms.gov/files/document/31820-cms-adult-elective-surgery-andprocedures-recommendations.pdf

Chiu, P. W. Y., Ng, S. C., Inoue, H., Reddy, D. N., Hu, E. L., Cho, J. Y., . . Rerknimitr, R. (2020). Practice of endoscopy during COVID-19 pandemic: position statements of the Asian Pacific Society for Digestive Endoscopy (APSDE-COVID statements). Gut, 69(6), 991-996.

Coccolini, F., Perrone, G., Chiarugi, M., Di Marzo, F., Ansaloni, L., Scandroglio, I., . . . Forfori, F. (2020). Surgery in COVID-19 patients: operational directives. World Journal of Emergency Surgery, 15, 1-7.

Collaborative, C. (2020). Global guidance for surgical care during the COVID-19 pandemic. Br J Surg. 
Diaz, A., Sarac, B. A., Schoenbrunner, A. R., Janis, J. E., \& Pawlik, T. M. (2020). Elective surgery in the time of COVID-19: Elsevier.

Elster, E., Potter, B. K., \& Chung, K. (2020). Response to COVID-19 by the surgical community. Surgery.

European Society for Medical Oncology (ESMO). (2020). COVID-19 and cancer. Retrieved May, 2020, from https://www.esmo.org/newsroom/covid-19-andcancer

Forrester, J. D., Nassar, A. K., Maggio, P. M., \& Hawn, M. T. (2020). Precautions for operating room team members during the COVID-19 pandemic. Journal of the American College of Surgeons.

Guerci, C., Maffioli, A., Bondurri, A. A., Ferrario, L., Lazzarin, F., \& Danelli, P. (2020). COVID-19: How can a department of general surgery survive in a pandemic? Surgery.

Iacobucci, G. (2020). Covid-19: all non-urgent elective surgery is suspended for at least three months in England: British Medical Journal Publishing Group.

Lei, S., Jiang, F., Su, W., Chen, C., Chen, J., Mei, W., . . Liu, D. (2020). Clinical characteristics and outcomes of patients undergoing surgeries during the incubation period of COVID-19 infection. EClinicalMedicine, 100331.

National Health Service (NHS). (2020). Speciality guide acute treatement of cancer. Retrieved May, 2020, from https://www.england.nhs.uk/coronavirus/wpcontent/uploads/sites/52/2020/03/specialty-guide-acutetreatment-cancer-23-march2020.pdf

National Institute for Health and Care Excellence. (2020, 29 April 2020). COVID-19 rapid guideline: critical care in adults. Retrieved May, 2020, from https://www.nice.org.uk/guidance/ng159

Patel, Z. M., Fernandez-Miranda, J., Hwang, P. H., Nayak, J. V., Dodd, R., Sajjadi, H., \& Jackler, R. K. (2020). Letter: Precautions for Endoscopic Transnasal Skull Base Surgery During the COVID-19 Pandemic. Neurosurgery. doi: 10.1093/neuros/nyaa125

Rajan, N., \& Joshi, G. P. (2020). COVID-19: Role of Ambulatory Surgery Facilities in This Global Pandemic. Anesthesia and analgesia.

Ross, S. W., Lauer, C. W., Miles, W. S., Green, J. M., Christmas, A. B., May, A. K., \& Matthews, B. D. (2020). Maximizing the calm before the storm: tiered surgical response plan for novel coronavirus (COVID-19). Journal of the American College of Surgeons.

Royal College of Surgeons of Edinburgh. (2020). Intercollegiate General Surgery Guidance on COVID-19 UPDATE. Retrieved May, 2020, from https://www.rcsed.ac.uk/newspublic-affairs/news/2020/march/intercollegiate-general-surgery-guidance-on-covid19-update

Royal College of Surgeons of England. (2020). COVID-19: Good Practice for Surgeons and Surgical Teams. Retrieved May, 2020, from https://www.rcseng.ac.uk/standardsand-research/standards-and-guidance/good-practice-guides/coronavirus/covid-19good-practice-for-surgeons-and-surgical-teams/

Society of American gastrointestinal and endoscopic surgeons (SAGES). (2020). SAGES AND EAES RECOMMENDATIONS REGARDING SURGICAL RESPONSE TO COVID-19 CRISIS. Retrieved May, 2020, from https://www.sages.org/recommendations-surgical-response-covid-19/

State guidance on elective surgeries. (2020). Retrieved May, 2020, from https://www.ascassociation.org/covid-19-state. 
Tan, T. (2004). How severe acute respiratory syndrome (SARS) affected the Department of Anaesthesia at Singapore General Hospital. Anaesthesia and intensive care, 32(3), 394-400.

Tan, Y.-t., Wang, J.-w., Zhao, K., Han, L., Zhang, H.-q., Niu, H.-q., . . Lei, T. (2020). Preliminary recommendations for surgical practice of neurosurgery department in the central epidemic area of 2019 coronavirus infection. Current Medical Science, 1-4.

Tompkins, B. M., \& Kerchberger, J. P. (2010). Personal protective equipment for care of pandemic influenza patients: A training workshop for the powered air purifying respirator. Anesthesia \& Analgesia, 111(4), 933-945.

Tuech, J.-J., Gangloff, A., Di Fiore, F., Michel, P., Brigand, C., Slim, K., . . Schwarz, L. (2020). Strategy for the practice of digestive and oncological surgery during the Covid-19 epidemic. Journal of visceral surgery.

Vukkadala, N., Qian, Z. J., Holsinger, F. C., Patel, Z. M., \& Rosenthal, E. (2020). COVID-19 and the otolaryngologist-preliminary evidence-based review. The Laryngoscope.

Wong, J., Goh, Q. Y., Tan, Z., Lie, S. A., Tay, Y. C., Ng, S. Y., \& Soh, C. R. (2020). Preparing for a COVID-19 pandemic: a review of operating room outbreak response measures in a large tertiary hospital in Singapore. Canadian Journal of Anesthesia/Journal canadien d'anesthésie, 1-14.

World Health Organization. (2020a). Coronavirus disease (COVID-2019) situation reports. Retrieved 15 May, 2020, from https://www.who.int/emergencies/diseases/novelcoronavirus-2019/situation-reports

World Health Organization. (2020b). WHO Director-General's opening remarks at the media briefing on COVID-19 - 16 March 2020. Retrieved March, 2020, from https://www.who.int/dg/speeches/detail/who-director-general-s-opening-remarks-atthe-media-briefing-on-covid-19---16-march-2020

Zheng, M. H., Boni, L., \& Fingerhut, A. (2020). Minimally invasive surgery and the novel coronavirus outbreak: lessons learned in China and Italy. Annals of surgery. 